\title{
Response to Chiasson et al.: Acarbose for the prevention of Type 2 diabetes, hypertension and cardiovascular disease in subjects with impaired glucose tolerance: facts and interpretations concerning the critical analysis of the STOP-NIDDM Trial data
}

Most regretfully, the response by Chiasson et al. to our For Debate article [1] has not clarified any of the critical points we made. In contrast, it confirms most of our objections with regard to the methodology and the results of the STOP-NIDDM Trial $[2,3,4,5]$. Chiasson et al. write: "...for patients with risk of cardiovascular events, the number of patients at risk with acarbose treatment is smaller than the number of patients at risk in the placebo group. This is due to the fact that premature discontinuation of study medication was more frequent in the acarbose group". What this means is that patients who discontinued the medication, mostly due to acarbose-induced side effects, were not included in the at-risk population. Even if some of the patients were only examined at the end of the trial, they should still have been included in the atrisk population for the life table analysis, but they were not. Hence, from the total group of 714 patients randomised to acarbose, 55 had no follow-up examination at all, and up to 211 patients who discontinued their participation in the trial early were not followedup regularly every 3 months like the rest of the patients, which is not in accordance with the intentionto-treat evaluation principle. Therefore, the number of patients at risk is much smaller in the acarbose group, which very well explains the difference of 17 patients with documented cardiovascular events between the groups.

Also, despite published correspondence on this issue [5], Chiasson et al. again fail to comment on the possible case of data suppression. We asked for an explanation of the fact that 61 patients were excluded from the efficacy analysis because of no post-randomisation data, but data on cardiovascular events in

Received: 13 April 2004 / Accepted: 17 April 2004

Published online: 19 May 2004

(C) Springer-Verlag 2004 these patients have been published by the sponsor of the trial on the Internet $[1,5]$. In addition, the authors report [4] adverse events in all 1429 patients (Tables 2 and 4 ). We do not understand how the authors were able to assess adverse events in all randomised patients during the course of the trial, but had no chance to document any post-randomisation data on cardiovascular events in at least 61 patients [3].

With regard to the predefinition of cardiovascular endpoints, we can only comment on published information $[2,3]$ because we have no access to unpublished "on file" protocols of this study. Clearly, endpoints have been changed from the publication of the study protocol [2] to the publication of the study results [3]. In the published protocol [2] secondary endpoints are myocardial infarction, cerebrovascular accident and congestive heart failure; in the publication of the results [3], three secondary endpoints were added: peripheral vascular disease, angina and revascularisation procedures. The authors report 17 nonpredefined secondary endpoint events in the acarbose group and 33 in the placebo group [3]. Chiasson et al. fail to inform us why these endpoints were subsequently added, or omitted in the publication of the study protocol [2], and whether the statistical comparison of the published predefined endpoints yields significant results. Most surprisingly, mortality, which was twice as high in the acarbose group as in the placebo group [4], was not regarded as a study endpoint.

In the published protocol [2], the final close-out visit was defined after a 3-month washout period on placebo. A life table analysis of the primary endpoint results including this final examination has never been published, nor has any amendment to the protocol explaining why the method of evaluation was changed.

In addition, Chiasson et al. do not explain the rationale behind assessing the risk for the development of hypertension in patients who are already hypertensive. 
The response by Chiasson et al. confirms the fact that the study was not double-blind. If acarbose and placebo were uptitrated until side effects developed, it must have been easy for the investigators to guess which patients were taking what. It is still unclear to us why, in the two STOP-NIDDM publications [3, 4] which were published only a year apart, blood pressure and weight were evaluated in different ways. As a rationale for the change in the study protocol regarding the definition of hypertension, Chiasson et al. quote a practice guideline [6]. However, this guideline was published a year after the first publication of the STOP-NIDDM study results [4] and 3 months after the second publication [3]. The reason for the refusal to give us the $\mathrm{HbA}_{1} \mathrm{c}$ and the 2-h OGTT data during the active phase of the trial is also not stated.

To confirm the results of the STOP-NIDDM Trial, Chiasson et al. refer to a recent meta-analysis by Hanefeld et al. on the effects of acarbose in Type 2 diabetes [7]. This meta-analysis includes seven studies selected by unknown methods. Two of the trials were not published at all and two were published as abstract only. In the remaining three fully published trials, one reported "no serious adverse events" and one "no change of vital signs" for both placebo and acarbose. The last trial reported one serious adverse event (heart failure) for acarbose and none for placebo. Published data therefore do not show any advantage of acarbose treatment concerning cardiac events. The data obviously stem from a Bayer database. The reason for not including the largest long-term trial of acarbose (UKPDS 44) is not given. The UKPDS 44 [8] investigated 1946 patients, while 2061 patients were pooled for the Bayer meta-analysis [7]. In the UKPDS 44, a similar risk of any diabetes-related endpoints including cardiovascular events was found in the two groups [8]. This meta-analysis suffers from the same main methodological drawback as the STOP-NIDDM Trial: the selective non-follow-up of patients randomised to acarbose. Immediately after randomisation, 155 patients in the acarbose group and 88 patients in the placebo group were excluded from the at-risk population [7].

We do not criticise the fact that Bayer employees actively participated in all parts of the trial including the Steering Committee and the STOP-NIDDM Trial Research Group [2], and that the STOP-NIDDM authors received honoraria from Bayer. However, withholding this information in the publication of results $[3,4]$ and declaring that there was no conflict of interest [3] is misleading.

In conclusion, both the STOP-NIDDM Trial and the meta-analysis of selected studies with acarbose are seriously flawed and no valid conclusions can be drawn from the published data.

P. T. Sawicki, T. Kaiser

DIeM-Institute for Evidence Based Medicine, Cologne, Germany

\section{References}

1. Kaiser T, Sawicki PT (2004) Acarbose for prevention of diabetes, hypertension and cardiovascular events? A critical analysis of the STOP-NIDDM data. Diabetologia 47:575-580

2. Chiasson J-L, Gomis R, Hanefeld M et al. (1998) The STOP-NIDDM Trial. An international study on the efficacy of an $\alpha$-glucosidase inhibitor to prevent type 2 diabetes in a population with impaired glucose tolerance: rationale, design, and preliminary screening data. Diabetes Care 21:1720-1725

3. Chiasson JL, Josse RG, Gomis R et al. (2003) Acarbose treatment and the risk of cardiovascular disease and hypertension in patients with impaired glucose tolerance. The STOP-NIDDM Trial. JAMA 290:486-494

4. Chiasson JL, Josse RG, Gomis R et al. (2002) Acarbose for prevention of type 2 diabetes mellitus: the STOP-NIDDM randomised trial. Lancet 359:2072-2077

5. Kaiser T, Sawicki PT (2003) Acarbose for patients with hypertension and impaired glucose tolerance. JAMA 290:3066

6. Cifkova R, Erdine S, Fagard R et al. (2003) Practice guidelines for primary care physicians: $2003 \mathrm{ESH} / \mathrm{ESC}$ hypertension guidelines. J Hypertens 21:1779-1786

7. Hanefeld M, Cagatay M, Petrowitsch T et al. (2004) Acarbose reduces the risk for myocardial infarction in type 2 diabetic patients: meta-analysis of seven long-term studies. Eur Heart J 25:10-16

8. Holman RR, Cull CA, Turner RC (1999) A randomized double-blind trial of acarbose in type 2 diabetes shows improved glycemic control over 3 years. Diabetes Care 22:960-964

P. T. Sawicki (

DIeM-Institute for Evidence Based Medicine,

Venloer Str. 301-303, 50823 Cologne, Germany

E-mail: peter.sawicki@t-online.de

Fax: +49-221-55911103 